\title{
Trisomy 18 in One of Fraternal Twins
}

\section{DENNIS GERTZER and GERALD NATHENSON}

\author{
From the Montefiore Hospital and Medical Center, New York 10467 and the Morrisania City Hospital,
} New York 10452, USA

In the 10 years since trisomy 18 was first described by Edwards et al (1960) there have been numerous single case reports, some of which include reviews of other cases, indicating the rarity of this syndrome (Finley, Finley, and Carter, 1963; Zellweger, Beck, and Hawtrey, 1964). The incidence has been estimated as $1: 4500$ (Conen and Erkman, 1966) to $1: 7000$ (Taylor, 1968) live births.

Recently at this hospital, twins were born, one of whom had trisomy 18. This unlikely occurrence has only been reported once before (Karunkaran and Pai, 1967).

\section{Case Reports}

$\mathrm{Mr}$ and Mrs J., parents of the patients, are negroes. The father was 39 years old, and the mother, para 4 gravida 5, was 30 years at the time of delivery. All previous pregnancies were uncomplicated, and produced full term singletons with birth weights ranging from $3232 \mathrm{~g}$ to $2268 \mathrm{~g}$.

Received 21 December 1970.
The maternal medical history was complicated by the appearance of a euthyroid goiter, noted during the first pregnancy. Therapy consisted of an unknown antithyroid medication taken before 1967, and desiccated thyroid taken intermittently until 1968 . The rest of the family history was unremarkable except for the youngest sib, who at 5 years of age is below the 3rd centile for length and head circumference and has developmental retardation in association with severe congenital deafness. There were no abortions or neonatal deaths.

The pregnancy was full term and apparently uncomplicated, with only one antenatal clinic visit one month before delivery. Amniotic membranes ruptured 2 days before delivery. The labour was normal.

Twin A, a girl (Fig. 1), was delivered without complication, but was apnoeic at birth with an Apgar score of 1. With resuscitative measures spontaneous respiras tions ensued. Examination revealed a weight of $1247 \mathrm{~g}$ head circumference $28 \mathrm{~cm}$, length $38 \mathrm{~cm}$, heart rat $120 / \mathrm{min}$, respiratory rate $60 / \mathrm{min}$. Her head had gaping fontanelles and occipital prominence. There were inferior corneal infiltrations with scarring, bilaterally. The ears were low set and malformed. There was bilateral 
cleft lip and palate. The neck was short and webbed. The left hemithorax was more prominent, but there was no heart murmur. There was no organomegaly on abdominal examination, although the kidneys were palpable bilaterally. External genitalia were normal. The fingers had flexion deformities with overlapping of the index fingers over the middle fingers. Later, neurological examination revealed an active neonate with a good cry, but poor suck.

During the next 10 days she required an atmosphere enriched with oxygen. She developed conjunctivitis and pneumonia, and finally died. Radiography revealed a thin calvarium, thin ribs, and cardiomegaly.

Necropsy examination demonstrated a normal brain both grossly and microscopically. There was bronchopneumonia and pulmonary congestion. Cardiac anomalies included a PDA, hypoplasia of the left heart and branches of the pulmonary arteries and aorta, a VSD and right heart dilation. There were no GU or GI abnormalities.

Twin B, a boy (Fig. 1), was born within minutes of twin A. He was vigorous after birth. His birth weight was $2730 \mathrm{~g}$, length $48 \mathrm{~cm}$, head circumference $34 \mathrm{~cm}$, chest circumference $32 \mathrm{~cm}$. His neonatal course was unremarkable. He has been followed closely and is growing along the 25th centile for length and weight. There was a significant increase in head circumference in the first two and a half months of life, rising from the 15th to 97th centile; then following this percentile to the present. Psychomotor development to date has been normal.

Mrs J. recently delivered a normal infant girl.

Chromosomal Studies. Cytogenetic studies were done on lymphocytes grown in culture by a modification of the micromethod of Arakaki and Sparkes (1963). Twin A had 18-trisomy (Fig. 2). Chromosomal studies on lymphocyte cultures of twin B (Fig. 2), the mother and deaf sib were normal. The father was unavailable for study.

\section{Discussion}

Comparison of these twin sibs dramatically demonstrated the devastating effect of autosomal trisomy on intrauterine growth. On clinical grounds alone, the actual diagnosis of trisomy 18 syndrome in this patient was somewhat difficult. Although the sex, low birth weight, occipital prominence, lowset malformed ears, webneck, congenital heart disease, flexion deformities of the fingers, and thin ribs seen on $x$-ray were all compatible with this diagnosis, the severe cleft lip and palate suggested trisomy 13. Taylor (1968) has quite clearly indicated the overlap of clinical findings in these syndromes, and the difficulty of establishing a definite diagnosis on clinical grounds alone. Cytogenetic studies are necessary to establish a specific diagnosis.

The likelihood of the event described in this report based on the incidence of fraternal twins in the non-white population $(1.01 \%)$ and the incidence of trisomy $18(1: 4500)$ is one in 450,000 live births.

The presence of different trisomic syndromes in sibs is known (Turner, den Dulk, and Watkins, 1964; Campbell, 1969), but there have been on reports associating trisomy with other congenital abnormalities in disomic siblings. This family contains one sibling (twin B) with relative macrocephaly (length and weight increasing along the 25th centile, head circumference along the 97th centile), and one sib with congenital deafness, microcephaly, and short stature. Both show normal chromosomal patterns by lymphocyte culture techniques. The significance of this finding is unclear.

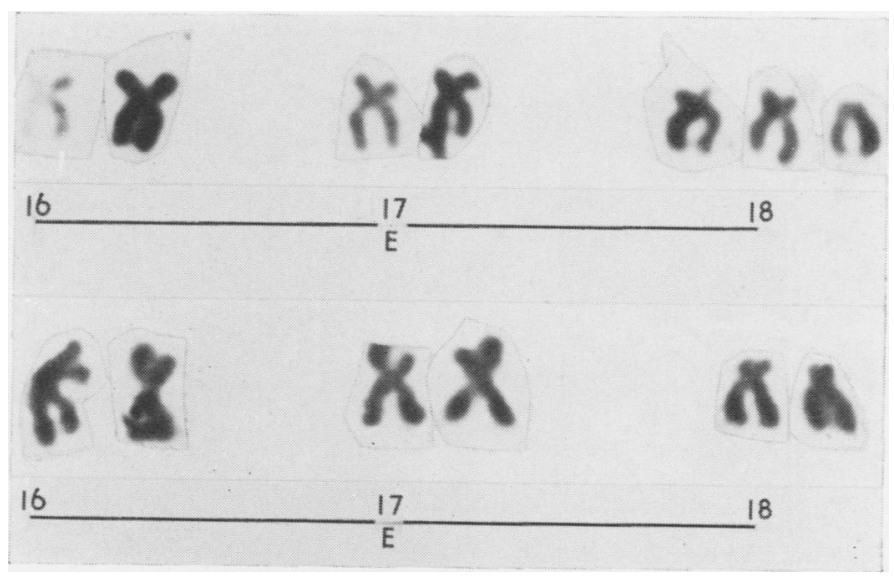

FIG. 2. E group from the karyotype of twin A (top) and twin B (bottom). 


\section{Summary}

Fraternal twins are described, one of whom had 18-trisomy. The likelihood of this occurrence is $1: 450,000$ live births.

\section{REFERENCES}

Arakaki, D. T. and Sparkes, R. S. (1963). Microtechnique for culturing leukocytes from whole blood. Cytogenetics, 2, 57-60.

Campbell, J. B. (1969). Abnormal siblings in patients with trisomy 13-15 and 16-18. Military Medicine, 134, 513-515.

Conen, P. E. and Erkman, B. (1966). Frequency and occurrence of chromosomal syndromes. II B-trisomy. American fournal of Human Genetics, 18, 387-398.
Edwards, J. H., Harnden, D. G., Cameron, A. H., Cross, V. M., and Wolf, O. H. (1960). A new trisomic syndrome. Lancet, 1, 787789.

Finley, W. H., Finley, S. C., and Carter, E. T. (1963). 17-18 trisomy Syndrome: review and report of a case. American fournal of Diseases of Children, 106, 591-596.

Karunakaran, E. K. and Pai, R. A. (1967). E trisomy syndrome in a dyzygotic twin. Indian Pediatrics, 4, 145-149.

Taylor, A. I. (1968). Autosomal Trisomy Syndromes: A detailed study of 27 cases of Edward's syndrome and 27 cases of Patau's syndrome. Fournal of Medical Genetics, 5, 227-252.

Turner, B., den Dulk, G. M., and Watkins, G. (1964). The 17-18 trisomy and 21 trisomy syndromes in siblings. Fournal of Pediatrics, 64, 601-604.

Zellweger, H., Beck, K., and Hawtrey, C. E. (1964). Trisomy 18: Report of a case and discussion of the syndrome. Archives of Internal Medicine, 113, 596-605. 